\title{
Controlling a Class of Hyperbolic Distributed Parameter Systems Producing Ideal Turbulence
}

\author{
Masayasu Suzuki, Jun-ichi Imura and Kazuyuki Aihara
}

\begin{abstract}
We try to stabilize steady solutions of physical models described by a class of first-order linear partial differential equations with nonlinear boundary conditions. These systems are distributed parameter systems in which ideal turbulence, introduced by Sharkovsky et al., occurs. In this report, using the method of characteristics, we design a control law to stabilize equilibrium solutions and synchronize a pair of the distributed parameter systems.
\end{abstract}

\section{INTRODUCTION}

Ideal turbulence, which is the notion introduced by Sharkovsky et al. [1], [2], is a complex phenomenon that occurs in distributed parameter systems (DPSs) induced, in particular, by boundary value problems for hyperbolic partial differential equations (PDEs). In systems having ideal turbulence, one can often observe cascade processes of emergence of structures of decreasing scales or processes that even lead to stochastization of the systems. From this viewpoint, ideal turbulence is considered to be a good notion to understand features of real turbulence mathematically.

Recently, controlling spatiotemporal chaos has attracted great interest. In fact, the dynamics of spatiotemporal systems is often quite complicated and produces rich patterns with respect to time and space, which cannot be captured by low-dimensional dynamics. Therefore, it is natural and important to extend approaches to controlling chaos of low-dimensional dynamics to the high-dimensional case. The investigations of controlling spatiotemporal chaos have been carried out in several aspects, e.g., controlling spatiotemporal chaos in coupled map lattices [3], synchronizing it in PDEs [4], anti-controlling (i.e. generating chaotic behavior) for systems described by hyperbolic PDEs [5], and so on. In [6], [7], for a class of physical models described by a linear wave equation with nonlinear boundary conditions, especially, for time-delayed Chua's circuits [1], we try to stabilize steady solutions, which mean equilibrium solutions and periodic solutions, and synchronize a pair of the circuits by using d'Alembert's solution.

The contribution in this paper is to extend the results for the linear wave equation systems in [7] to that for systems described by first-order linear hyperbolic PDEs

This research is partially supported by Aihara Project, the FIRST program from JSPS.

M. Suzuki is with the FIRST Aihara Innovative Mathematical Modelling Project, JST, Japan ma-suzuki@ieee.org

J. Imura is with the Department of Mechanical and Environmental Informatics, Graduate School of Information Science and Engineering, Tokyo Institute of Technology, Tokyo, Japan imura@mei.titech.ac.jp

$\mathrm{K}$. Aihara is with the Institute of Industrial Science, University of Tokyo, Tokyo, Japan He is also with the FIRST Aihara Innovative Mathematical Modelling Project, JST, Japan aihara@sat.t.u-tokyo.ac.jp whose coefficients depend on spatial coordinate. To this end, we use the method of characteristics. The method of characteristics is a geometric solution method for a certain class of DPSs, especially, first-order hyperbolic PDE systems [8]. The feature of hyperbolic PDEs is the existence of infinitedimensional modes of nearly the same amount of energy; hence, it cannot be accurately represented by a finite number of modes. On the other hand, the method of characteristics is a strong tool for hyperbolic PDEs, since this method does not include any approximation such as discretization. Actually, in chemical engineering, the method of characteristics has been used for designing control law to develop the performance of the processes [9]-[11]. Furthermore, in literature [12][14], boundary conditions to stabilize equilibria in hyperbolic PDE systems have been investigated by using the method of characteristics with Riemann coordinates. Our technique is similar to [12]-[14] in the sense of designing a controller at the boundary. It should, however, be stressed that we consider the PDE systems having nonlinear boundary conditions, which generate complex phenomena like ideal turbulence. Focusing on the nonlinear structure caused by the boundary conditions and using chaos control techniques (e.g., [15]), we design control laws not only to globally stabilize the equilibrium solutions but also synchronize such systems.

\section{IDEAL TURBULENCE AND THE METHOD OF CHARACTERISTICS}

Ideal turbulence is a spatio-temporal vibration phenomenon which has quite intricate geometrical structure after the time evolution. The mathematical definition is given by Sharkovsky [1]: for an initial state, if the $\omega$-limit set of it contains at least a fractal function, or at least a random function, then the initial state is said to generate ideal turbulence, or stochastic ideal turbulence, respectively. In this section, an example of systems in which ideal turbulence occurs is introduced.

\section{A. Time-delayed Chua's circuit}

Let us consider a lossless transmission line with Chua's diode [16] as shown in Fig. 1(a).

Initial and boundary value problem (IBVP): The transmission line is denoted by a wave equation

$$
\left\{\begin{array}{ll}
L & \partial_{t} i+\partial_{x} v=0 \\
C & \partial_{t} v+\partial_{x} i=0
\end{array}, x \in[-l, l], t \in \mathbb{R}_{+},\right.
$$

and has boundary conditions

$$
\begin{aligned}
& v(-l, t)=0, \quad t \in \mathbb{R}_{+}, \\
& i(l, t)=G(v(l, t)-R i(l, t)-E), t \in \mathbb{R}_{+},
\end{aligned}
$$




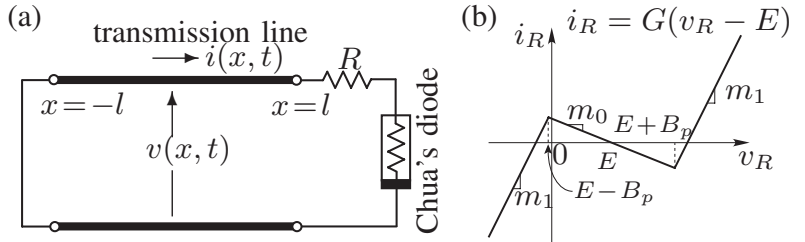

Fig. 1. (a) Lossless transmission line with Chua's diode; (b) The voltagecurrent characteristic of Chua's diode; Here $v_{R}$ and $i_{R}$ are the voltage and the current, applied to the diode, respectively.

where $E$ denotes a bias voltage, and $G$ represents the voltage-current characteristic of Chua's diode given as follows (see Fig. 1(b)).

$$
G(\xi)=m_{1} \xi+\frac{1}{2}\left(m_{0}-m_{1}\right)\left[\left|\xi+B_{p}\right|-\left|\xi-B_{p}\right|\right]
$$

For the wave equation (1), by mean of a variable transformation

$$
v=p-q, i=(p+q) / Z,
$$

we derive PDEs whose new variables $p, q$ are separated.

$$
\left\{\begin{array}{c}
\partial_{t} p+\nu \partial_{x} p=0 \\
\partial_{t} q-\nu \partial_{x} q=0
\end{array}, x \in[-l, l], t \in \mathbb{R}_{+} .\right.
$$

Here, $Z$ and $\nu$ are the characteristic impedance and the propagation velocity of the transmission line, and given by $Z=\sqrt{L / C}$ and $\nu=1 / \sqrt{L C}$, respectively.

In general, first-order PDEs are solvable by using the method of characteristics. For (4), introducing parameters $r$, $s \in \mathbb{R}$ and integrating the following coupled ordinary differential equations (ODEs) with appropriate initial conditions

$$
\begin{aligned}
& \frac{d t}{d r}=1, \frac{d x}{d r}=\nu, \frac{d p}{d r}=0 \\
& \frac{d t}{d s}=1, \frac{d x}{d s}=-\nu, \frac{d q}{d r}=0
\end{aligned}
$$

we can find solutions of the PDE (4). ODEs (5) and (6) have trajectories each of which takes constant values along lines $t-x / \nu=$ const. and lines $t+x / \nu=$ const., respectively. Hence, the general solutions of Eq. (4) can be represented by using some functions $\alpha, \beta:[0, \infty) \rightarrow \mathbb{R}$ as follows.

$$
p(x, t)=\alpha(t-(x-l) / \nu), q(x, t)=\beta(t+(x+l) / \nu) .
$$

Then, we have

$\left\{\begin{array}{l}v(x, t)=\alpha(t-(x-l) / \nu)-\beta(t+(x+l) / \nu), \\ i(x, t)=\frac{1}{Z}[\alpha(t-(x-l) / \nu)+\beta(t+(x+l) / \nu)] .\end{array}\right.$

Substituting (7) for the boundary conditions (2) and (3), we get a difference equation (DE) with continuous argument:

$$
\left\{\begin{array}{l}
\alpha(t+2 l / \nu)=\beta(t) \\
\beta(t+2 l / \nu)=f(\alpha(t))
\end{array}\right.
$$

where $f$ is described as follows.

$$
\begin{aligned}
& f(\eta)= \begin{cases}A_{1} \eta-B_{-1}, & \eta-\frac{E}{2}<-\delta, \\
A_{0} \eta-B_{0}, & \left|\eta-\frac{E}{2}\right| \leq \delta, \\
A_{1} \eta-B_{1}, & \eta-\frac{E}{2}>\delta,\end{cases} \\
& A_{0}=\frac{m_{0}(Z-R)-1}{m_{0}(Z+R)+1}, \quad B_{0}=\frac{m_{0} E Z}{m_{0}(Z+R)+1}, \\
& A_{1}=\frac{m_{1}(Z-R)-1}{m_{1}(Z+R)+1}, B_{-1}=\frac{\left[m_{1} E-\left(m_{1}-m_{0}\right) B_{p}\right] Z}{m_{1}(Z+R)+1}, \\
& B_{1}=\frac{\left[m_{1} E+\left(m_{1}-m_{0}\right) B_{p}\right] Z}{m_{1}(Z+R)+1}, \delta=\frac{\left[m_{0}(Z+R)+1\right] B_{p}}{2} \text {. }
\end{aligned}
$$

Eliminating $\alpha$ from Eqs. (8), we obtain

$$
\beta(t+4 l / \nu)=f(\beta(t)), t \geq 0 .
$$

Initial conditions $v(x, 0)=\varphi(x), i(x, 0)=\psi(x)$ for the IBVP give an initial condition $\beta(\tau), \tau \in[0,4 l / \nu]$ for DE (10) as follows.

$$
\begin{aligned}
& \beta((x+l) / \nu)=[-\varphi(x)+Z \psi(x)] / 2, x \in[-l, l], \\
& \beta(-(x-3 l) / \nu)=[\varphi(x)+Z \psi(x)] / 2, x \in[-l, l] .
\end{aligned}
$$

Then, the solution for (11) is uniquely determined by (10): $\beta(\tau)=f^{n}(\beta(\tau-4 n l / \nu)), 4 n l / \nu \leq \tau<4(n+1) l / \nu, n \in \mathbb{N}$,

which provides the corresponding solution for the IBVP as follows:

$$
\begin{aligned}
& \left(\begin{array}{c}
v(x, t) \\
i(x, t)
\end{array}\right)=\left(\begin{array}{c}
f^{\lambda}(\beta(\mu))-f^{\lambda+1}(\beta(\mu)) \\
f^{\lambda}(\beta(\mu))+f^{\lambda+1}(\beta(\mu)) / Z
\end{array}\right), \\
& \lambda \in \mathbb{N} \text { s.t. } \frac{\nu}{4 l}\left(t-\frac{x}{\nu}\right)-\frac{1}{4}<\lambda \leq \frac{\nu}{4 l}\left(t-\frac{x}{\nu}\right)+\frac{3}{4} \\
& \mu=t-\frac{x}{\nu}-\frac{(4 \lambda-3) l}{\nu} \in\left[0, \frac{4 l}{\nu}\right] .
\end{aligned}
$$

Thus, the time evolution of the distributed parameter system can be described by one-dimensional map $f$. Then we can investigate various properties of the original system, including the asymptotic behavior of it, by the map $f$. Sharkovsky et al. have proven the following theorem [1].

Theorem 1 (i) There occurs ideal turbulence if the map $f$ has an attracting periodic orbit with period $m>2$.

(ii) There occurs stochastic ideal turbulence if the map $f$ has no attracting periodic orbits.

Set the parameters of the circuit to $m_{0}=-1.5[1 / \mathrm{k} \Omega]$, $m_{1}=20[1 / \mathrm{k} \Omega], Z=0.51[\mathrm{k} \Omega], 2 l / \nu=400[\mathrm{~ns}], R=$ $0.05[\mathrm{k} \Omega], B_{p}=3.5[\mathrm{~V}]$ and $E=3[\mathrm{~V}]$. Then $f$ does not have any stable periodic orbit and the behavior becomes chaotic. Therefore, stochastic ideal turbulence occurs. Figure 2 shows the time evolution of the distribution of the voltage for timedelayed Chua's circuit with the above parameters, where a smooth initial state transfers to stochastic ideal turbulence.

\section{Stabilizing AN EQuilibrium Solution}

\section{A. System description}

Let us consider an initial and boundary-value problem of first-order linear PDEs with input $u(t)$ at a boundary $x=-l$ 


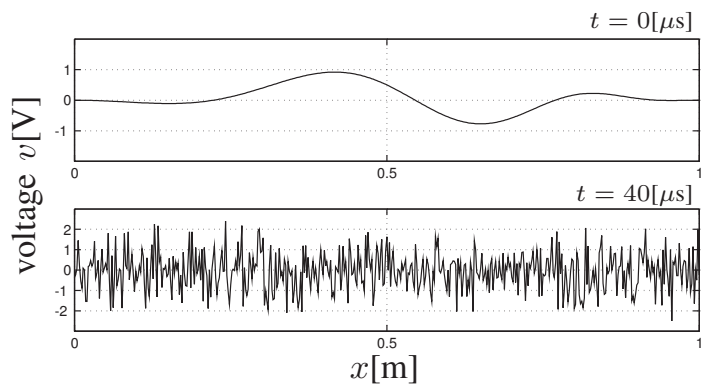

Fig. 2. Stochastic ideal turbulence: This figure shows the time development of the voltage distribution in the transmission line.

as follows.

$$
\left\{\begin{array}{l}
\partial_{t} p+a(x) \partial_{x} p=0, x \in[-l, l] \\
\partial_{t} q-a(x) \partial_{x} q=0, x \in[-l, l] \\
H_{1}(p(-l, t), q(-l, t), p(l, t), q(l, t), u(t))=0 \\
H_{2}(p(-l, t), q(-l, t), p(l, t), q(l, t), u(t))=0 \\
p(x, 0)=\varphi(x) \\
q(x, 0)=\psi(x)
\end{array}\right.
$$

Here, $a$ is a scalar function satisfying that $a(x)>0, x \in$ $[-l, l]$, and $H_{1}, H_{2}$ are boundary conditions. Moreover, we assume that we can observe the boundary values $p(l, t)$ and $q(-l, t)$ (i.e., the incoming-flow values into the right and the left boundaries, respectively) as the output of the system.

For ordinary differential equations

$$
d x / d t=a(x), \quad d x / d t=-a(x),
$$

denote the flows as

$$
\phi_{a}: \mathbb{R}_{+} \times[-l, l] \rightarrow[-l, l], \phi_{-a}: \mathbb{R}_{+} \times[-l, l] \rightarrow[-l, l] .
$$

Then, according to the method of characteristics, variables $p$ and $q$ in the first and second equations in IBVP (12) take constant values along these flows in the region $(-l, l)$. For the flow $\phi_{a}$, the time interval $T$, which is taken to reach to the boundary $x=l$ from the opposite boundary $x=-l$, is a root of an equation with respect to $t$ :

$$
l=\phi_{a}(t,-l) .
$$

Due to the monotonicity of the flow $(a(x)>0)$, this is uniquely determined. In a similar way, the time interval taken for the flow $\phi_{-a}$ to reach to $x=-l$ from $x=l$ is also $T$. Therefore, it turns out that

$$
p(-l, t)=p(l, t+T), \quad q(l, t)=q(-l, t+T) .
$$

Substituting the above representation for the boundary conditions in (12), we have

$$
\left\{\begin{array}{l}
H_{1}(p(l, t+T), q(-l, t), p(l, t), q(-l, t+T), u(t))=0 \\
H_{2}(p(l, t+T), q(-l, t), p(l, t), q(-l, t+T), u(t))=0 .
\end{array}\right.
$$

Now we consider the following assumption for IBVP (12).

Assumption 1 The above simultaneous equations can be solved with respect to $p(l, t+T)$ and $q(-l, t+T)$, and written by continuous functions $\hat{H}$ and $\check{H}$ as follows.

$$
\left\{\begin{array}{l}
\alpha(t+T)=\hat{H}(\alpha(t), \beta(t), u(t)), \\
\beta(t+T)=\check{H}(\alpha(t), \beta(t), u(t)) .
\end{array}\right.
$$

Here, for the sake of simplicity, we use $\alpha(t)=p(l, t)$ and $\beta(t)=q(-l, t)$.

(13) is a difference equation with continuous argument. There exists a lot of nonlinear boundary conditions satisfying this assumption: e.g., when the boundary conditions $H_{1}, H_{2}$ are defined by piecewise affine functions such as Chua's diode, the form (13) can be derived.

From the initial functions $\varphi, \psi$ for (12), we have sets

$I_{\alpha}:=\{\alpha(t) \mid 0 \leq t \leq T\}=\left\{\varphi\left(\phi_{a}(-t, l)\right) \mid 0 \leq t \leq T\right\}$,

$I_{\beta}:=\{\beta(t) \mid 0 \leq t \leq T\}=\left\{\psi\left(\phi_{-a}(-t,-l)\right) \mid 0 \leq t \leq T\right\}$,

as initial sets for the difference equation (13). For all time $t$, the values $\alpha(t)$ and $\beta(t)$, i.e., the values at the boundaries, $p(l, t)$ and $q(-l, t)$, are calculated by (13) with these initial sets. Meanwhile, the states in the inner of the region $[-l, l], p(x, t)$ and $q(x, t)$, are uniquely determined from the boundary-values. This is because these states take constant values along the flows $\phi_{a}$ and $\phi_{-a}$.

Let us consider a two-dimensional discrete-time dynamical system

$$
\left\{\begin{array}{l}
\alpha_{n+1}=\hat{H}\left(\alpha_{n}, \beta_{n}, u_{n}\right), \\
\beta_{n+1}=\check{H}\left(\alpha_{n}, \beta_{n}, u_{n}\right),
\end{array}\right.
$$

which corresponds to (13). In the following sections, we try to design a control law to stabilize an equilibrium solution of (12) using (14).

\section{B. Equilibrium solutions and the stability}

For solutions of $(12),\left(\begin{array}{ll}p & q\end{array}\right)^{\mathrm{T}}:[-l, l] \times \mathbb{R}_{+} \rightarrow$ $\mathbb{R}^{2}$, which take temporally-constant distributed-values, that is, solutions $\left(\begin{array}{lll}p^{*} & q^{*}\end{array}\right)^{\mathrm{T}}$ satisfying that $\left(p^{*} q^{*}\right)^{\mathrm{T}}(x, t)=$ $\left(p^{*} q^{*}\right)^{\mathrm{T}}(x, 0), x \in[-l, l]$ for all $t \geq 0$, let us call such solutions equilibrium solutions. For an equilibrium point of the discrete-time system (14), $\left(\alpha^{*} \beta^{*}\right)^{\mathrm{T}} \in \mathbb{R}^{2}$, we find that a pair of functions given by

$$
p^{*}(x, t) \equiv \alpha^{*}, q^{*}(x, t) \equiv \beta^{*},
$$

is an equilibrium solution for Eq. (12) without input.

Now, define a norm $\|\cdot\|$ on the function space $C^{0}\left([-l, l], \mathbb{R}^{2}\right)$ by

$$
\left\|(p q)^{\mathrm{T}}\right\|=\sup _{x \in[-l, l]}(|p(x)|+|q(x)|) .
$$

Using this norm, we give the definition of the stability of the equilibrium solution as follows.

Definition 1 An equilibrium solution $r^{*}=\left(p^{*} q^{*}\right)^{\mathrm{T}}$ of the system (12) is said to be stable if, for all $\varepsilon>0$, there exists a $\delta>0$ such that, for any solutions satisfying $\|(p q)^{\mathrm{T}}(\cdot, 0)-$ $r^{*} \|<\delta$, we have $\left\|(p q)^{\mathrm{T}}(\cdot, t)-r^{*}\right\|<\varepsilon$ for all $t>0$.

Definition 2 An equilibrium solution $r^{*}=\left(\begin{array}{ll}p^{*} q^{*}\end{array}\right)^{\mathrm{T}}$ of the system (12) is said to be globally asymptotically stable 
if it is stable and, for any initial condition $r$, we have $\lim _{t \rightarrow \infty}\left\|(p q)^{\mathrm{T}}(\cdot, t)-r^{*}\right\|=0$.

\section{Stabilizing the equilibrium solution}

In this section, we tackle the following problem. Problem: For the system (12), design a control law

$$
u(t)=u(p(l, t), q(-l, t))
$$

that stabilizes an equilibrium solution $\left(p^{*} q^{*}\right)^{\mathrm{T}}$ of (12) by using only the boundary values.

The basic idea is not to design a control law for controlling the state $\left(\begin{array}{ll}p & q\end{array}\right)^{\mathrm{T}}$ directly, but to keep $\alpha(t)$ and $\beta(t)$ in the neighborhood of $\alpha^{*}$ and $\beta^{*}$, respectively. At first, for (14), we design a control law to converge $\left(\alpha_{n}, \beta_{n}\right)$ to $\left(\alpha^{*}, \beta^{*}\right)$, and then, apply the control law to the original system (12). The following two propositions guarantee the validity of this strategy.

Proposition 1 Assume that, for the discrete-time system (14) there exists a state feedback law $u_{n}=\bar{u}\left(\alpha_{n}, \beta_{n}\right)$ such that, for the equilibrium point $\left(\alpha^{*} \beta^{*}\right)^{\mathrm{T}}, \bar{u}$ locally stabilizes $\left(\alpha^{*} \beta^{*}\right)^{\mathrm{T}}$ (that is, for all $\varepsilon>0$, there exist $a \delta>0$ and a state feedback input $\left\{u_{n}=\bar{u}\left(\alpha_{n}, \beta_{n}\right)\right\}$ such that, if $\left|\left(\alpha_{0} \beta_{0}\right)^{\mathrm{T}}-\left(\alpha^{*} \beta^{*}\right)^{\mathrm{T}}\right|<\delta$, we have $\mid\left(\alpha_{n} \beta_{n}\right)^{\mathrm{T}}-$ $\left(\alpha^{*} \beta^{*}\right)^{\mathrm{T}} \mid<\varepsilon$ for all $n>0$.). Then, using this control law $\bar{u}$ for the IBVP (12) as follows,

$$
u(t):=\bar{u}(\alpha(t), \beta(t))=\bar{u}(p(l, t), q(-l, t)),
$$

the equilibrium solution $\left(p^{*}, q^{*}\right)^{\mathrm{T}}$ of the IBVP is locally stabilized.

Proposition 2 If the equilibrium point of (14) can be globally asymptotically stabilized by a state feedback law $u_{n}=$ $\bar{u}\left(\alpha_{n}, \beta_{n}\right)$, then, by letting $u(t)=\bar{u}(\alpha(t), \beta(t))$, the equilibrium solution of the IBVP (12) can be globally asymptotically stabilized.

Proofs are given in Secs. A and B.

Remark 1 For many low-dimensional discrete-time chaotic systems, it is possible to globally stabilize equilibrium points by conventional chaos control schemes [17]. From the proposition, if an IBVP induces such a low-dimensional system to which existing control methods can be applied, then, for the IBVP, we can also use the control methods as boundary inputs. Therefore, one finds that the class of IBVPs such that their equilibrium solutions can be globally stabilized is large.

D. Applications: Stabilization of an equilibrium solution of time-delayed Chua's circuit with an inhomogeneous transmission line

For time-delayed Chua's circuit introduced in Sec. II-A, consider stabilizing an equilibrium solution of the system with control input at the boundary $x=-l$. In this section, assume that the parameters of the transmission line of timedelayed Chua's circuit depend on the space coordinate $x$, that is, $C(x)$ and $L(x)$, and the characteristic impedance

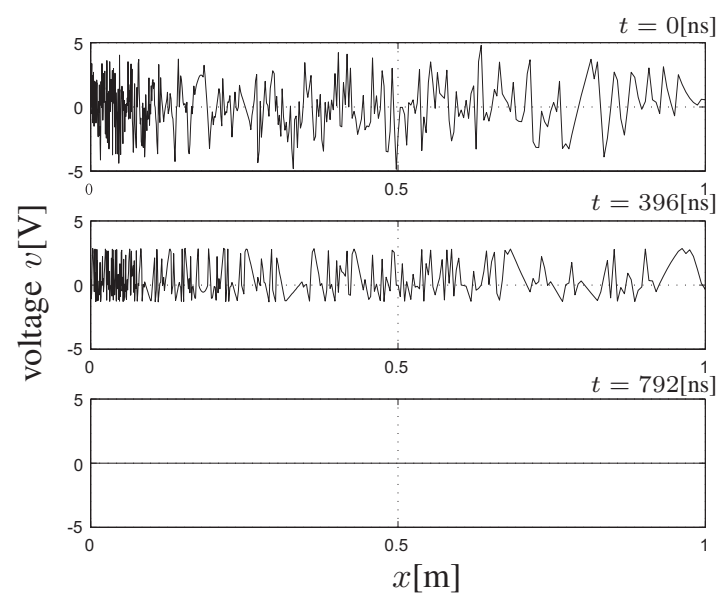

Fig. 3. Controlling stochastic ideal turbulence in an inhomogeneous transmission line: Stabilising an equilibrium solution.

however takes a constant value (does not depend on $x$, i.e., $Z=\sqrt{L(x) / C(x)}=$ const.). Then, using the same variable transformation as Sec.II-A, we can describe this system as (12). Here, $a(x)=1 / \sqrt{C(x) L(x)}$. The voltage and current signals propagate with non-constant velocity in the transmission line.

Let the boundary condition at $x=-l$ be

$$
v(-l, t)=u(t), \quad t \in \mathbb{R}_{+} .
$$

Then we get the following difference equation with continuous argument, where $f$ is defined by Eq. (9).

$$
\left\{\begin{array}{l}
\alpha(t+2 l / \nu)=\beta(t)+u(t) \\
\beta(t+2 l / \nu)=f(\alpha(t))
\end{array}, t \geq 0 .\right.
$$

The discrete-time system

$$
\left\{\begin{array}{l}
\alpha_{n+1}=\beta_{n}+u_{n} \\
\beta_{n+1}=f\left(\alpha_{n}\right)
\end{array}, n=0,1, \cdots\right.
$$

has an equilibrium point $\left(\alpha^{*}, \beta^{*}\right)=\left(B_{0} /\left(1-A_{0}\right), B_{0} /(1-\right.$ $\left.A_{0}\right)$ ). For (17), a state feedback law

$$
u_{n}=\bar{u}\left(\beta_{n}\right)=-\beta_{n}+\beta^{*},
$$

make the equilibrium point globally asymptotically stable. Therefore, for (16), by using the corresponding control law

$$
u(t)=\bar{u}(\beta(t))=-\beta(t)+\beta^{*},
$$

the equilibrium solution of the IBVP can be globally asymptotically stabilized. In fact, the variable $\beta(t)$ is represented as $\beta(t)=(-v(-l, t)+Z i(-l, t)) / 2$. Hence, the input can be given only by the boundary states, and written as follows.

$$
u(t)=[v(-l, t)-Z i(-l, t)] / 2+\beta^{*}, \quad t \in \mathbb{R}_{+} .
$$

Fig. 3 shows a simulation result using time-delayed Chua's circuit with an inhomogeneous transmission line, where we set $C(x)=1 /(10 x+11)[\mathrm{nF} / \mathrm{m}]$ and $L(x)=0.26 /(10 x+$ 11) $[\mathrm{mH} / \mathrm{m}]$. From Fig. 3, we can see that a complicated initial state converges to the equilibrium solution. 


\section{SYNCHRONIZATION}

We show that two identical hyperbolic PDE systems can be synchronized by using the same strategy mentioned in Secs. III. Consider two identical systems except for inputs. Define them as a master $\Sigma$ and a slave $\widetilde{\Sigma}$ by

$$
\begin{aligned}
& \Sigma:\left\{\begin{array}{l}
\partial_{t} p+a(x) \partial_{x} p=0, x \in[-l, l], \\
\partial_{t} q-a(x) \partial_{x} q=0, x \in[-l, l], \\
H_{1}(p(-l, t), q(-l, t), p(l, t), q(l, t), 0)=0, \\
H_{2}(p(-l, t), q(-l, t), p(l, t), q(l, t), 0)=0,
\end{array}\right. \\
& \tilde{\Sigma}:\left\{\begin{array}{l}
\partial_{t} \tilde{p}+a(x) \partial_{x} \tilde{p}=0, x \in[-l, l], \\
\partial_{t} \tilde{q}-a(x) \partial_{x} \tilde{q}=0, x \in[-l, l], \\
H_{1}(\tilde{p}(-l, t), \tilde{q}(-l, t), \tilde{p}(l, t), \tilde{q}(l, t), u(t))=0, \\
H_{2}(\tilde{p}(-l, t), \tilde{q}(-l, t), \tilde{p}(l, t), \tilde{q}(l, t), u(t))=0 .
\end{array}\right.
\end{aligned}
$$

Assume that each system can be reduced to a difference equation with continuous argument in the same way as Sec.III as follows.

$\Sigma:\left(\begin{array}{c}\alpha(t+T) \\ \beta(t+T)\end{array}\right)=\left(\begin{array}{c}\hat{H}(\alpha(t), \beta(t)) \\ \check{H}(\alpha(t), \beta(t))\end{array}\right)=: F(\alpha(t), \beta(t))$,

$\tilde{\Sigma}:\left(\begin{array}{c}\tilde{\alpha}(t+T) \\ \tilde{\beta}(t+T)\end{array}\right)=F(\tilde{\alpha}(t), \tilde{\beta}(t))+B u(t)$.

Consider discrete-time systems corresponding to the above difference equations:

$$
\begin{aligned}
& \left(\alpha_{n+1} \beta_{n+1}\right)^{\mathrm{T}}=F\left(\alpha_{n}, \beta_{n}\right), \\
& \left(\tilde{\alpha}_{n+1} \tilde{\beta}_{n+1}\right)^{\mathrm{T}}=F\left(\tilde{\alpha}_{n}, \tilde{\beta}_{n}\right)+B u_{n} .
\end{aligned}
$$

Now, we design a control law to synchronize these discretetime systems (19), (20) based on [15]. At first, we evaluate the error between the states of two systems by using an appropriate function $h$ as follows.

$$
D_{n}:=h\left(\tilde{\alpha}_{n}, \tilde{\beta}_{n}\right)-h\left(\alpha_{n}, \beta_{n}\right) .
$$

Next, we give a control law using a constant gain $K$ :

$$
u_{n}=K D_{n}=K\left[h\left(\tilde{\alpha}_{n}, \tilde{\beta}_{n}\right)-h\left(\alpha_{n}, \beta_{n}\right)\right] .
$$

If $F+B K h$ is a contractive map, then the systems (19), (20) can be synchronized.

For the original systems $\Sigma, \tilde{\Sigma}$, we give a control law using the above $K$ and $h$ as follows.

$$
u(t)=K[h(\tilde{\alpha}(t), \tilde{\beta}(t))-h(\alpha(t), \beta(t))] .
$$

\section{A. An application to time-delayed Chua's circuits}

In the rest of this paper, we try to synchronize a pair of identical time-delayed Chua's circuits with the inhomogeneous transmission lines:

$$
\begin{aligned}
& \Sigma:\left\{\begin{array}{l}
L(x) \partial_{t} i+\partial_{x} v=0, C(x) \partial_{t} v+\partial_{x} i=0 \\
v(-l, t)=0, \\
i(l, t)=G(v(l, t)-R i(l, t)-E)
\end{array}\right. \\
& \widetilde{\Sigma}:\left\{\begin{array}{l}
L(x) \partial_{t} \tilde{i}+\partial_{x} \tilde{v}=0, C(x) \partial_{t} \tilde{v}+\partial_{x} \tilde{i}=0 \\
\tilde{v}(-l, t)=0, \\
\tilde{i}(l, t)=G(\tilde{v}(l, t)-R \tilde{i}(l, t)-E+u(t))+w(t) .
\end{array}\right.
\end{aligned}
$$

Here, $u(t)$ and $w(t)$ represent a voltage input and a current input, respectively, and $C(x)=1 /(10 x+11)[\mathrm{nF} / \mathrm{m}], L(x)=$
$0.26 /(10 x+11)[\mathrm{mH} / \mathrm{m}]$. . Thus, in this section, we consider that the inputs of slave $\widetilde{\Sigma}$ are given at the boundary $x=l$, and show that the control strategy used for the stabilization of the equilibrium solution can be also used even if the circuit has control ports at $x=l$. Using the method of characteristics and letting $u(t)=(Z+R) w(t)$, we derive difference equations as follows.

$$
\begin{aligned}
& (\alpha(t+2 l / \nu) \beta(t+2 l / \nu))^{\mathrm{T}}=(\beta(t) f(\alpha(t)))^{\mathrm{T}} \\
& (\tilde{\alpha}(t+2 l / \nu) \tilde{\beta}(t+2 l / \nu))^{\mathrm{T}}=\left(\tilde{\beta}(t) f(\tilde{\alpha}(t))+\frac{Z}{Z+R} u(t)\right)^{\mathrm{T}}
\end{aligned}
$$

Consider discrete-time systems corresponding to the above difference equations:

$$
\begin{aligned}
& \left(\alpha_{n+1} \beta_{n+1}\right)^{\mathrm{T}}=\left(\beta_{n} f\left(\alpha_{n}\right)\right)^{\mathrm{T}} \\
& \left(\tilde{\alpha}_{n+1} \tilde{\beta}_{n+1}\right)^{\mathrm{T}}=\left(\tilde{\beta}_{n} f\left(\tilde{\alpha}_{n}\right)\right)^{\mathrm{T}}+\left(0 \frac{Z}{Z+R}\right)^{\mathrm{T}} u_{n}
\end{aligned}
$$

and give the input $u_{n}$ by

$$
u_{n}=K\left(h\left(\tilde{\alpha}_{n}\right)-h\left(\alpha_{n}\right)\right) .
$$

Letting $K=(Z+R) / Z$ and choosing the function $h$ as

$h(\eta)= \begin{cases}\left(A_{0}-a\right) \delta, & \eta \leq-\delta+E / 2, \\ \left(a-A_{0}\right)(\eta-E / 2), & -\delta+E / 2<\eta<\delta+E / 2, \\ \left(a-A_{0}\right) \delta, & \eta \geq \delta+E / 2,\end{cases}$

where $a$ is a constant number that is less than 1 , the function $f+\frac{Z}{Z+R} \cdot K h$ becomes a contraction map. Hence, the systems (22) and (23) eventually synchronize. Finally, for the slave $\widetilde{\Sigma}$, by letting

$$
\begin{aligned}
u(t) & =K(h(\tilde{\alpha}(t))-h(\alpha(t))) \\
& =\frac{Z+R}{Z}\left[h\left(\frac{\tilde{v}(l, t)+Z \tilde{i}(l, t)}{2}\right)-h\left(\frac{v(l, t)+Z i(l, t)}{2}\right)\right],
\end{aligned}
$$

the distributed voltages and currents in transmission lines of $\Sigma$ and $\hat{\Sigma}$ can be synchronized. The top and third figures in Fig. 4 show initial states and states after 3960[ns], respectively. Moreover, the second and the fourth figures show the difference between them. Comparing the bottom figure with the second one, it can be seen that the states are synchronizing although the initial states are quite different.

\section{Conclusions}

In this report, for a class of infinite-dimensional dynamical systems described by a first-order linear PDE with nonlinear boundary conditions, a control method to stabilize equilibrium solutions has been proposed by using the method of characteristics. Even if ideal turbulence occurs in such a system, it has been confirmed that the stabilization is accomplished only by boundary inputs. We have also applied the proposed method to time-delayed Chua's circuits, and shown by numerical simulation that equilibrium solutions are stabilized and two identical circuits can be synchronized. Thus, this paper extended the control method for a linear wave equation systems in [7] to that for first-order linear hyperbolic PDE systems whose coefficients depend on spatial coordinate. 

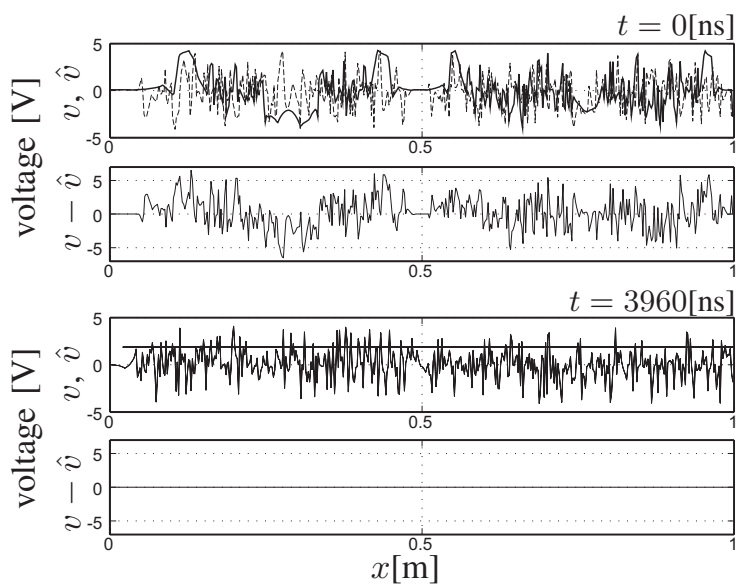

Fig. 4. Synchronization of two time-delayed Chua's circuits: In the top and the third figures, solid lines and broken lines mean $v(x, l)$ and $\hat{v}(x, l)$, respectively; The second and fourth figures show the difference between the master and slave.

\section{ACKNOWLEDGMENTS}

This research is partially supported by Aihara Innovative Mathematical Modelling Project, the Japan Society for the Promotion of Science (JSPS) through its "Funding Program for World-Leading Innovative R\&D on Science and Technology (FIRST Program)."

\section{REFERENCES}

[1] A. N. Sharkovsky, "Ideal turbulence," Nonlinear Dynamics, vol. 44, pp. $15-27,2006$.

[2] E. Y. Romanenko and A. N. Sharkovskii, "Dynamical systems and simulation of turbulence," Ukrainian Mathematical Journal, vol. 59, no. 2, pp. 229-242, 2007.

[3] G. Hu and Z. Qu, "Controlling spatiotemporal chaos in coupled map lattice systems," Phys. Rev. Lett., vol. 72, pp. 68-71, 1994.

[4] L. Kocarev, Z. Tasev, and U. Parlitz, "Synchronizing spatiotemporal chaos of partial differential equations," Phys. Rev. Lett., vol. 79, no. 1, pp. 51-54, 1997.

[5] Y. Huang, "Boundary feedback anticontrol of spatiotemporal chaos for 1D hyperbolic dynamical systems," Int. J. Bifurcation and Chaos, vol. 14, no. 5, pp. 1705-1723, 2004.

[6] M. Suzuki and N. Sakamoto, "Controlling ideal turbulence and an application to communications," in The 48th IEEE Conference on Decision and Control, 2009.

[7] _ - "Controlling ideal turbulence in time-delayed chua's circuit: Stabilization and synchronization," Int. J. Bifurcation and Chaos, vol. 20, no. 5, pp. 1351-1363, 2010.

[8] V. I. Arnold, Geometrical Methods in the Theory of Ordinary Differential Equations. Springer, 1988.

[9] H. Sira-Ramirez, "Distributed sliding mode control in systems described by quasilinear partial differential equations," System \& Control Letters, vol. 13, pp. 177-181, 1989.

[10] H. Shang, J. F. Forbes, and M. Guay, "Feedback control of hyperbolic distributed parameter systems," Chemical Engineering Science, vol. 60, pp. 696-980, 2005.

[11] E. Aggelogiannaki and H. Sarimveis, "Robust nonlinear $H_{\infty}$ control of hyperbolic distributed parameter systems," Control Engineering Practice, vol. 17, pp. 723-732, 2009.

[12] J. M. Greenberg and L. T. Tsien, "The effect of boundary damping for the quasilinear wave equations," J. Diffrential Equations, vol. 52, pp. 66-75, 1984.

[13] J.-M. Coron, B. d'Andréa, and G. Bastin, "A strict Lyapunov function for boundary control of hyperbolic systems of conservation laws," IEEE Trans. on Automatic Control, vol. 52, no. 1, pp. 2-11, 2007.

[14] C. Prieur, J. Winkin, and G. Bastin, "Robust boundary control of systems of conservation laws," Mathematics of Control, Signals and Systems, vol. 20, pp. 173-197, 2008.
[15] T. Ushio, "Chaotic synchronization and controlling chaos based on contraction mappings," Phys. Lett. A, vol. 198, pp. 14-22, 1995.

[16] L. O. Chua, "Chua's circuit 10 years later," Int. J. of Circuit Theory and Applications, vol. 22, pp. 279-305, 1994.

[17] S. Boccaletti, C. Grebogi, Y. C. Lai, H. Mancini, and D. Maza, "The control of chaos: theory and applications," Physics Reports, vol. 329, pp. 103-197, 2000.

\section{APPENDIX}

\section{A. A proof of Proposition 1}

Define the $\varepsilon$-neighborhood around a point $\left(y_{1} y_{2}\right)^{\mathrm{T}}$ in $\mathbb{R}^{2}$ by $V_{\varepsilon}\left(y_{1}, y_{2}\right)=\left\{\left(\begin{array}{ll}z_{1} & z_{2}\end{array}\right)^{\mathrm{T}}||\left(\begin{array}{ll}z_{1} & z_{2}\end{array}\right)^{\mathrm{T}}-\left(\begin{array}{ll}y_{1} & y_{2}\end{array}\right)^{\mathrm{T}} \mid<\varepsilon\right\}$. If the initial sets of (13) satisfy ${ }^{1}\left\{(\alpha(\tau) \beta(\tau))^{\mathrm{T}}\right\}_{\tau \in[0, T)} \subset$ $V_{\delta}\left(\alpha^{*}, \beta^{*}\right)$, then, under the control law $\bar{u}$, it turns out that $\left\{(\alpha(n T+\tau) \beta(n T+\tau))^{\mathrm{T}}\right\}_{\tau \in[0, T)} \subset V_{\varepsilon}\left(\alpha^{*}, \beta^{*}\right), n=1,2, \cdots$. Therefore, for all $t \geq 0$, we have $\left\{(\alpha(t) \quad \beta(t))^{\mathrm{T}}\right\}_{t>0} \subset$ $V_{\varepsilon}\left(\alpha^{*}, \beta^{*}\right)$.

While we have confirmed that the states at boundaries of (12) keep taking values in the neighborhood of $\left(\alpha^{*} \beta^{*}\right)^{\mathrm{T}}$, the states of the interior of the distributed system are considered as below. Reviewing the ordinary differential equation $d x / d t=a(x)$, we find that the time $\kappa$, which a state $x \in[-l, l]$ spends to reach to the boundary $l$, one-to-one corresponds to the state $x$ due to the monotonicity(, where $\left.0 \leq \kappa \leq t_{1}\right)$. From this viewpoint, we can define a map $T_{a}: x \mapsto T_{a}(x)=\kappa$. Using the method of characteristics, we find that $p(x, t)=p\left(l, t+T_{a}(x)\right)=\alpha\left(t+T_{a}(x)\right)$. In a similar way, for the equation $d x / d t=-a(x)$, by defining a map $T_{-a}$ giving the cost time which a state $x \in[-l, l]$ spends to reach to the boundary $-l$, we have $q(x, t)=q\left(-l, t+T_{-a}(x)\right)=\beta\left(t+T_{-a}(x)\right)$. Consequently, it turns out that, for all $t$,

$$
\begin{aligned}
& \left\|(p q)^{\mathrm{T}}(\cdot, t)-\left(p^{*} q^{*}\right)^{\mathrm{T}}\right\|=\sup _{x \in[-l, l]}\left(p(x, t)-p^{*}|+| q(x, t)-q^{*} \mid\right) \\
& \leq \sup _{x \in[-l, l]}\left|\alpha\left(t+T_{a}(x)\right)-\alpha^{*}\right|+\sup _{x \in[-l, l]}\left|\beta\left(t+T_{-a}(x)\right)-\beta^{*}\right|<2 \varepsilon .
\end{aligned}
$$

\section{B. A proof of Proposition 2}

The local stability of the equilibrium solution is guaranteed by Proposition 1. Here, we confirm that each trajectory with an arbitrary initial condition converges to the equilibrium solution. From the global asymptotically stability of (14), for each element $\left(\alpha_{0}, \beta_{0}\right)$ in the initial sets and all $\varepsilon>0$ there exists a $N=N\left(\left(\alpha_{0}, \beta_{0}\right), \varepsilon\right)<\infty$ such that $\mid\left(\alpha_{n} \beta_{n}\right)^{\mathrm{T}}-$ $\left(\alpha^{*} \beta^{*}\right)^{\mathrm{T}} \mid<\varepsilon$ for all $n \geq N$. Since the initial sets are bounded, there exists a maximal value of $N, \widetilde{N}$, when one fixes $\varepsilon$ and varies the value $\left(\alpha_{0}, \beta_{0}\right)$ in the initial sets. Therefore, we have $\left|(\alpha(t) \beta(t))^{\mathrm{T}}-\left(\alpha^{*} \beta^{*}\right)^{\mathrm{T}}\right|<\varepsilon, t \geq \widetilde{N} T$. Consequently, it turns out that

$$
\begin{aligned}
& \left\|(p q)^{\mathrm{T}}(\cdot, t)-\left(p^{*} q^{*}\right)^{\mathrm{T}}\right\| \\
& \leq \sup _{x \in[-l, l]}\left|\alpha\left(t+T_{a}(x)\right)-\alpha^{*}\right|+\sup _{x \in[-l, l]}\left|\beta\left(t+T_{-a}(x)\right)-\beta^{*}\right| \\
& \rightarrow 0 \quad(\text { as } \quad t \rightarrow \infty),
\end{aligned}
$$

and hence, the equilibrium solution of (12) is globally asymptotically stable.

\footnotetext{
${ }^{1}$ Actually, it is possible when the initial function $(\varphi \psi)^{\mathrm{T}}$ of (12) is enough close to the equilibrium solution $\left(p^{*} q^{*}\right)^{\mathrm{T}}$.
} 